\title{
Estimate of the neutral atoms' contribution to the Mercury exosphere caused by a new flux of micrometeoroids
}

\author{
P. Borin ${ }^{1}$, M. Bruno ${ }^{2}$, G. Cremonese ${ }^{3}$, and F. Marzari ${ }^{4}$ \\ ${ }^{1}$ CISAS, University of Padova, via Venezia 15, 35131 Padova, Italy \\ e-mail: patrizia.borin@unipd.it \\ 2 Department of Mineralogical and Petrological Science, via Valperga Caluso 35, 10125, Torino, Italy \\ e-mail: marco.bruno@unito.it \\ 3 INAF-Astronomical Observatory of Padova, Vicolo dell'Osservatorio 5, 35131 Padova, Italy \\ e-mail: gabriele.cremonese@oapd.inaf.it \\ 4 Department of Physics, via Marzolo 8, 35131 Padova, Italy \\ e-mail: marzari@pd.infn.it
}

Received 24 February 2010 / Accepted 7 May 2010

\begin{abstract}
Context. The planet Mercury has an extended and tenuous exosphere made up of atoms that are ejected from the surface by energetic processes, including hypervelocity micrometeoritic impacts, photon-stimulated desorption by UV radiation, and ion sputtering. The well known constituents of the Hermean exosphere are $\mathrm{H}, \mathrm{He}, \mathrm{O}, \mathrm{Na}, \mathrm{K}$, and $\mathrm{Ca}$ but, from the new MESSENGER data from flybys, many others elements are expected, as for instance $\mathrm{Mg}$.

Aims. Meteoroid impacts are an important source of neutral atoms in the exosphere of Mercury. We estimate the vapor and neutral atom production rates on Mercury caused by impacts of micrometeoroids of sizes between 5-100 $\mu \mathrm{m}$. The micrometeoritic flux is derived from a new statistical approach based on direct numerical integrations of dust particle trajectories under the action of the Poynting-Robertson drag and the gravitational attraction of all planets.

Methods. We included two different calibration sources for the meteoroid flux in our calculations of the vapor and neutral atoms and also considered both asteroidal and cometary sources for the dust. Three different surface compositions, which might be found on the planet, have been adopted, each with a different mass fraction of atoms in the regolith of the planet.

Results. We derive different values of neutral atom vapor production rates assuming different calibration sources for the meteoroid flux. The three simple mineralogical surface compositions show significant differences in the related production rates, and they are all greater than those reported in the previous papers assuming other dominant source mechanisms. Our neutral atom production rates are about one order of magnitude higher than the previous estimates. This implies that the impact vaporization has a much higher contribution than previously assumed.
\end{abstract}

Key words. planets and satellites: atmospheres - methods: statistical

\section{Introduction}

Mercury has an extended and tenuous exosphere made up of atoms that are released from the surface by energetic processes. These processes are generally considered to be micrometeoritic impacts, photon-stimulated desorption by UV radiation, ion sputtering and thermal vaporization. It is known that Mercury's exosphere contains $\mathrm{H}, \mathrm{He}$ and $\mathrm{O}$, discovered by Mariner 10, and $\mathrm{Na}, \mathrm{K}$ and $\mathrm{Ca}$ seen by ground-based telescopes (Bida et al. 2000; Killen \& Ip 1999; Killen et al. 2005; Potter \& Morgan 1985; Potter \& Morgan 1986). Moreover, Mg was observed by the MESSENGER MASCS instrument during the second Mercury flyby (McClintock et al. 2009; Killen et al. 2009). The Mg emission appears to be uniformly distributed, but it shows a weak north-south enhancement. Observations made during the first Mercury flyby of MESSENGER instead revealed a distinct north-south asymmetry of the Na tail and a strong dawndusk asymmetry in the night-side Ca distribution. In addition, $\mathrm{Ca}$ emission is strong near the equatorial regions and decreases toward higher latitudes, while Na emission has an high-latitude enhancement relative to the equatorial regions. Signals from both $\mathrm{Mg}$ and $\mathrm{Ca}$ are weaker than that of $\mathrm{Na}$ (McClintock et al. 2009).

Photon stimulated and thermal desorption are mechanisms that can explain the concentrations and temperature of $\mathrm{Na}$ and $\mathrm{K}$ in the exosphere. Chemical sputtering and solar wind sputtering are suggested to be responsible for the release of the metal atoms and water vapor (Potter 1995). Together with the ion sputtering, the meteoroid impacts are considered one of the main sources of $\mathrm{Ca}$ in the exosphere (Killen et al. 2005).

According to Killen et al. (2001) impact vaporization is responsible of only about a quarter of the flux of the $\mathrm{Na}$ forming the exosphere. However, on the night side of the planet meteoroid bombardment is a much more significant source because ion sputtering is active only on the day side of Mercury. An estimate of the production rate of $\mathrm{Na}$ caused by micrometeoroid impacts is given by Hunten et al. (1988) suggesting a value of of $10^{22} \mathrm{Na} \mathrm{s}^{-1}$. Morgan et al. (1988) give instead an orbital average vaporization rate of about $0.1514 \times 10^{23} \mathrm{Na} \mathrm{s}^{-1}$. Leblanc and Johnson (2003) in their model assume a value of about $5 \times 10^{23} \mathrm{Na} \mathrm{s}^{-1}$ at perihelion. Cremonese et al. (2005) suggest that meteoroids with a radius extending $10^{-1} \mathrm{~m}$ do not significantly 
contribute to the daily observations of the exosphere because of their low impact probability on Mercury's surface. The total mass of large meteoroids hitting the planet account at most for $3 \%$ of the vapor mass produced also taking into account that not all mass of the projectiles contributes to the vapor. Large meteoroids, which have an impact frequency of approximately two events/year, may produce only a localized and temporary increase in the exospheric density (Mangano et al. 2007).

We calculate the vapor and neutral atoms production rates on Mercury, caused by the impacts of micrometeoroids. We consider the size range between $5-100 \mu \mathrm{m}$ and adopt the impactor flux estimates given by the new dynamical model of Borin et al. (2009, hereafter BM). In this size range, dust particles are significantly perturbed by Poynting-Robertson and solar wind drag and spiral towards the Sun on timescales that depend on their size and composition. According to Bruno et al. (2007) and Cremonese et al. (2005) the production of neutral $\mathrm{Na}$ atoms is mainly due to meteoroids larger than $10^{-5} \mathrm{~m}$. As a consequence, approximately $50 \%$ of the Na comes from impacting meteoroids in the size range $5 \times 10^{-6}-10^{-4} \mathrm{~m}, 43 \%$ from $10^{-4}-10^{-3} \mathrm{~m}$, $6 \%$ from $10^{-3}-10^{-2} \mathrm{~m}$ and the remaining $1 \%$ from meteoroids with a size larger than $1 \mathrm{~cm}$. Cintala (1992) also states that $76 \%$ of all vapor is generated by meteoroids with masses in between $10^{-3}-10^{-7} \mathrm{~g}$ (radius range $10^{-4}-10^{-5} \mathrm{~m}$ ), and that projectiles with a radius greater than $10^{-3} \mathrm{~m}$ would have a negligible effect on the vapor production.

The calculations presented here were performed with two different calibration sources for the meteoroid flux. The first is based on the data from Love \& Brownlee (1993) (as done in $\mathrm{BM}$ ), while the second is based on data by Grün et al. (1985), hereafter LB and $\mathrm{G}$ respectively. Moreover, we give different values of the vapor production rates, taking into account the different balance between asteroidal and cometary sources of dust particles (Wiegert et al. 2009; Dermott et al. 2002). We consider three types of surface composition with different mass fraction of atoms in the regolith of the planet (Cremonese et al. 2005; Goettel 1988; Smith \& Marconi 1995).

\section{Vapor production}

The volume of target material vaporized by a spherical particle of mass $m$ and impacting velocity $v$ can be estimated with the relation given by Cintala (1992)

$V_{\text {vap }}(v, m)=\frac{m}{\varrho_{\mathrm{p}}}\left(c+d v+e v^{2}\right)$,

where $m$ and $\varrho_{\mathrm{p}}=2.5 \frac{\mathrm{g}}{\mathrm{cm}^{3}}$ are the mass and the density of the meteoroid that impacts the surface, $c$ is a constant, $d$ and $e$ are given in $\mathrm{km}^{-1} \mathrm{~s}$ and $\mathrm{km}^{-2} \mathrm{~s}^{2}$ and depend on target temperature and projectile composition (Cintala 1992).

Assuming that the vapor composition is determined by the target composition, the production rate (atoms $\mathrm{cm}^{-2} \mathrm{~s}^{-1}$ ) of neutral atoms $S$ is calculated by the following relation (Morgan \& Killen 1997)

$S_{i}=M_{\mathrm{vap}}\left(\frac{f_{i}}{p_{i}}\right) N_{\mathrm{A}}$,

where $M_{\text {vap }}$ is the vapor production rate $\left(\mathrm{g} \mathrm{cm}^{-2} \mathrm{~s}^{-1}\right), p_{i}$ is the atomic weight of the atom, $N_{\mathrm{A}}$ is the Avogadro's number and $f_{i}$ is the mass fraction of the neutral atom on the surface. It is possible to calculate $M_{\text {vap }}$ integrating the following equation

$M_{\mathrm{vap}}=\varrho_{t} \int_{v_{\min }}^{v_{\max }} \int_{m_{\min }}^{m_{\max }} \phi(v, m) V_{\mathrm{vap}}(v, m) \mathrm{d} v \mathrm{~d} m$, where $\varrho_{\mathrm{t}}=1.8 \frac{\mathrm{g}}{\mathrm{cm}^{3}}$ is the target density, $\phi(v, m)$ is the differential number of impacts as a function of the meteoroid velocity and radius, $v_{\min }=0 \mathrm{~km} \mathrm{~s}^{-1}, v_{\max }=80 \mathrm{~km} \mathrm{~s}^{-1}, m_{\min }=1.309 \times 10^{-9} \mathrm{~g}$, $m_{\max }=1.047 \times 10^{-5} \mathrm{~g}$.

To compare our results with those reported in Cintala (1992) we rewrite the equations used by the author to compute the vapor production rate of neutral atoms. The differential flux can be written as

$\phi(v, m)=f(v) \cdot h(m)$,

where $f(v)$ is the velocity distribution of dust particles $(\mathrm{s} / \mathrm{km})$ and $h(m)$ is the mass distribution function of the impacting particles $\left(\mathrm{g}^{-1} \mathrm{~cm}^{-2} \mathrm{~s}^{-1}\right)$. The velocity distribution function is given by the equation

$f_{\mathrm{C}}(v)=k r^{1.5}\left[\frac{v}{\sqrt{r\left(v^{2}-v_{\mathrm{Me}}^{2}\right)+v_{\mathrm{Ee}}^{2}}}\right]^{3} \mathrm{e}^{\left(-\xi \sqrt{r\left(v^{2}-v_{\mathrm{Me}}^{2}\right)+v_{\mathrm{Ee}}^{2}}\right)}$,

where $k=3.81, \xi=0.247$ are constants, $r=0.387 \mathrm{AU}$ is the mean distance of Mercury from the Sun, $v$ is the impact velocity of dust particles on Mercury, $v_{\mathrm{Ee}}=11.1 \mathrm{~km} \mathrm{~s}^{-1}$ is the escape velocity for the Earth at $100 \mathrm{~km}$ altitude, $v_{\mathrm{Me}}=4.25 \mathrm{~km} \mathrm{~s}^{-1}$ is the escape velocity at the surface of Mercury. The Cintala's mass distribution is

$h_{\mathrm{C}}(m)=-\frac{1}{m F_{1}} \exp \left(\sum_{i=0}^{11} c_{i} \ln (m)^{i}\right) \cdot\left[\sum_{i=1}^{11} i \cdot c_{i} \ln (m)^{i-1}\right]$,

where $F_{1}=0.364, c_{i}$ are constants (Cintala 1992; Cremonese et al. 2005).

Following BM we can compute the neutral atoms and the vapor production rates from the previous relations ( 2 and 3 ), while the velocity distribution function is given by the equation

$f_{\mathrm{B}}(v)=\sqrt{\frac{2}{\pi}} a^{-\frac{3}{2}} v^{2} \exp \left(-\frac{v^{2}}{2 a}\right) \cdot b$,

where $a=106.3$ and $b=6,8616 \times 10^{4}$ are constants. The derived mass distribution function is given by

$h_{\mathrm{B}-\mathrm{LB}}(m)=(0.045 \pm 0.015) \cdot h_{\mathrm{C}}(m)$,

where $h_{\mathrm{B}-\mathrm{LB}}(m)$ is a fit of the data obtained by means of simulations used by BM to compute the flux with Love and Brownlee calibration. Finally

$h_{\mathrm{B}-\mathrm{G}}(m)=(0.025 \pm 0.005) \cdot h_{\mathrm{C}}(m)$,

where $h_{\mathrm{B}-\mathrm{G}}(m)$ is a fit of the data obtained by means of simulations used by BM to compute the flux with the Grün calibration.

\section{Mercury surface composition}

We note that a deeper knowledge of the Mercury's surface composition is fundamental for obtaining a reliable estimate of the production rate of neutral atoms. Unfortunately, the mineralogical composition of the planet is still poorly constrained by ground based observations (Sprague et al. 2009) and MESSENGERS's data. As a consequence, our estimates of $S_{i}$, which strongly depends on the surface composition through the coefficients $f_{i}$, could be substantially revised when a more detailed knowledge of the Mercury's mineralogy will be gained. In any case, our model allows to derive the $S_{i}$ values for any surface composition by simply inserting the different estimates of 
P. Borin et al.: Estimate of the neutral atoms' contribution to the Mercury exosphere caused by a new flux of micrometeoroids

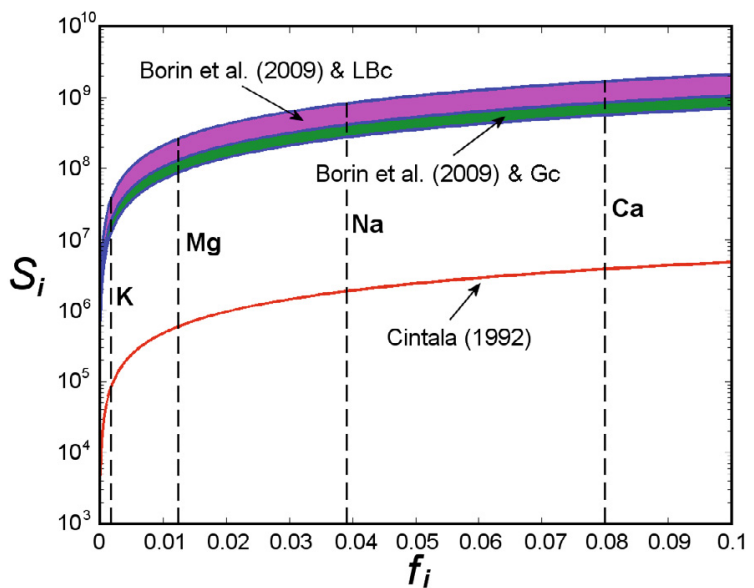

Fig. 1. Production rate $S_{i}$ (Number of atoms $\mathrm{cm}^{-2} \mathrm{~s}^{-1}$ ) with different weight percentages of atoms in the surface of the planet $f_{i}$.

Table 1. Mass fraction of elements in the regolith attended in the surface of Mercury.

\begin{tabular}{cccc}
\hline \hline Mass Fraction & Cremonese et al. & Goettel & Smith and Marconi \\
\hline$f_{\mathrm{Na}}$ & 0.0380 & 0.0044 & 0.0033 \\
$f_{\mathrm{K}}$ & 0.0013 & 0.0003 & - \\
$f_{\mathrm{Ca}}$ & 0.0797 & 0.0375 & - \\
$f_{\mathrm{Mg}}$ & 0.0123 & 0.211 & - \\
\hline
\end{tabular}

References. (1) Cremonese et al. (2005); (2) Goettel (1988); (3) Smith \& Marconi (1995).

$f_{i}$ values into Eq. (2) (Fig. 1). As a consequence, the proposed model is a valid tool to estimate the neutral elements production caused by the meteoritic impact on the Mercury's surface.

We consider three simple surface mineralogical compositions of Mercury which allow us to estimate the neutral atoms release and to show how it may change for different $f_{i}$. The first one is the same as that in Cremonese et al. (2005), where the surface is assumed to be spatially homogeneous and made up of regolith with an anorthositic composition (90\% plagioclase and $10 \%$ pyroxene in volume). They consider as a first approximation that the surface of Mercury is made up only of anorthosite, which seems to be a rock extensively distributed on the surface (e.g., Blewett et al. 2002; Sprague et al. 2000; Sprague et al. 2002; and Warell 2003). The second one is that given by Goettel (1988), which presents a refractory-rich model, a volatile-rich model, and three moderately refractory-rich models. In this work we use the Goettel second model (adding K) in our computations and we take as mass fraction a mean value of the lower and upper limits reported by the author in his work.

The third model is taken from Smith and Marconi (1988), but only for the Na mass fraction.

Table 1 reports the mass fraction of atoms in the surface of Mercury as adopted in Cremonese et al. (2005) and Bruno et al. (2007), by Goettel (1988) and by Smith and Marconi (1995).

\section{Calibration of the model}

The micrometeoritic flux computed by BM is obtained by a statistical approach based on numerical integrations of the orbits of dust particles under the action of the Poynting-Robertson drag and the gravitational attraction of all the planets. The numerical flux, derived by simulations following the evolution of 1000 particles with initial orbital elements typical of dust coming from
Table 2. Micrometeoritic flux obtained by Cintala and BM model with Love and Brownlee and Grün calibration in the range 5-100 $\mu \mathrm{m}$.

\begin{tabular}{ccc}
\hline \hline Model & $\frac{N}{\text { year }}$ & $\frac{g}{\mathrm{~cm}^{2} \mathrm{~s}}$ \\
\hline Cintala's model & $4.073 \times 10^{16}$ & $1.402 \times 10^{-16}$ \\
Borin model (LB calibration) & $3.104 \times 10^{18}$ & $2.382 \times 10^{-14}$ \\
Borin model (G calibration) & $4.596 \times 10^{17}$ & $8.982 \times 10^{-15}$ \\
\hline
\end{tabular}

Table 3. Asteroidal and cometary percentages contributing to the flux given by Dermott et al. (2002) and Wiegert et al. (2009).

\begin{tabular}{ccc}
\hline \hline Source & Asteroidal contribution & Cometary contribution \\
\hline Dermott et al. (2002) & $66 \%$ & $34 \%$ \\
Wiegert et al. (2009) & $4 \%$ & $96 \%$ \\
\hline
\end{tabular}

References. (1) Dermott et al. (2002); (2) Wiegert et al. (2009).

the Main Belt, has to be calibrated on real flux data. This calibration is performed with the flux data on the Earth of Love \& Brownlee (1993), derived from the satellite Long Duration Exposure Facility (LDEF). We also consider a different calibration based on Grün et al. (1985). They compute the interplanetary flux at 1 AU from measurements of in situ experiments on board of HEOS 2 and Pioneers 8 and 9.

Table 2 shows the computed micrometeoritic flux measured in $N /$ year (where $N$ is the number of impacts) and $\mathrm{g} /\left(\mathrm{cm}^{2} \mathrm{~s}\right.$ ) given by Cintala, and by BM using LB and $\mathrm{G}$ calibration. It is possible to see that the LB calibration, is about seven (given in $\mathrm{N} /$ year) and three (given in $\mathrm{g} / \mathrm{cm}^{2} \mathrm{~s}$ ) times higher than the $\mathrm{G}$ calibration.

According to some authors (Dermott et al. 2002), the dust collected from satellite LDEF (Love \& Brownlee 1993) comes from typical asteroidal orbits, but a recent model of dust claims that a fraction as high as $90 \%$ might come from both short and long period comets (Wiegert et al. 2009). The flux values of BM reported in Table 2 were obtained by considering dust reaching the Earth on orbits initially placed on the inner edge of the asteroid belt with low values of eccentricity and inclination. Actually, the measured flux of dust, like that obtained by LDEF (Love \& Brownlee 1993), might consist of two components distinguishable only on the basis of their impact velocity. One component approaches the Earth on low eccentricity and low inclination orbits as those modelled by BM and it is possibly coming from the asteroid belt and some short period comets (Dermott et al. 2002). The second component, characterized by high impact velocities, would originate from comets with high values of eccentricity and inclination (Wiegert et al. 2009). The flux estimate given by $\mathrm{BM}$ is relative to the first component and a tuning coefficient is needed to evaluate the flux on Mercury derived by this component once calibrated to the LDEF data.

\section{Results}

In this section we report the values of vapor and neutral atoms production rates obtained by considering

- LB and G calibrations;

- the percentages of cometary and asteroidal components expected to come from low eccentricity and low inclination orbits (Dermott et al. 2002; Wiegert et al. 2009);

- the surface compositions provided by Cremonese et al. (2005); Goettel (1988) and Smith and Marconi (1995). 
Table 4. Comparison of the production rate $S_{i}$ of released elements caused by micrometeoritic impacts.

\begin{tabular}{cc|cc|cc}
\hline \hline & Cintala & \multicolumn{2}{c}{ Dermott et al. } & \multicolumn{2}{c}{ Wiegert et al. } \\
& & BM-LB & BM-G & BM-LB & BM-G \\
\hline $\mathrm{Na}$ & $9.1000 \times 10^{5}$ & $3.9417 \times 10^{8}$ & $2.2008 \times 10^{8}$ & $2.3889 \times 10^{7}$ & $1.338 \times 10^{7}$ \\
$\mathrm{~K}$ & $1.8305 \times 10^{4}$ & $7.9291 \times 10^{6}$ & $4.4271 \times 10^{6}$ & $4.8055 \times 10^{5}$ & $2.6831 \times 10^{5}$ \\
$\mathrm{Mg}$ & $2.7861 \times 10^{5}$ & $1.2068 \times 10^{8}$ & $6.7381 \times 10^{7}$ & $7.3141 \times 10^{6}$ & $4.0837 \times 10^{6}$ \\
$\mathrm{Ca}$ & $1.0948 \times 10^{6}$ & $4.7423 \times 10^{8}$ & $2.6478 \times 10^{8}$ & $2.8741 \times 10^{7}$ & $1.6047 \times 10^{7}$ \\
\hline
\end{tabular}

Notes. Here we consider the estimate of the flux given by Cintala (1992) tuned by the fraction of dust of asteroidal origin given by Dermott et al. (2002) and Wiegert et al. (2009), respectively. The assumed mineralogical surface composition follows from Cremonese et al. (2005). $S_{i}$ is given in $N \mathrm{~cm}^{-2} \mathrm{~s}^{-1}$.

References. (1) Cintala (1992); (2) Dermott et al. (2002); (3) Cremonese et al. (2005); (4) Wiegert et al. (2009).

Table 5. Same as Table 1 but for the mineralogical surface composition given by Goettel (1988). $S_{i}$ is given in $N \mathrm{~cm}^{-2} \mathrm{~s}^{-1}$.

\begin{tabular}{cc|cc|cc}
\hline \hline & Cintala & \multicolumn{2}{c}{ Dermott et al. } & \multicolumn{2}{c}{ Wiegert et al. } \\
& & BM-LB & BM-G & BM-LB & BM-G \\
\hline $\mathrm{Na}$ & $1.0537 \times 10^{5}$ & $4.5641 \times 10^{7}$ & $2.5483 \times 10^{7}$ & $2.7661 \times 10^{6}$ & $1.5444 \times 10^{6}$ \\
$\mathrm{~K}$ & $4.2243 \times 10^{3}$ & $1.8298 \times 10^{6}$ & $1.0216 \times 10^{6}$ & $1.1090 \times 10^{5}$ & $6.1917 \times 10^{4}$ \\
$\mathrm{Mg}$ & $4.7795 \times 10^{6}$ & $2.0702 \times 10^{9}$ & $1.1559 \times 10^{9}$ & $1.2547 \times 10^{8}$ & $7.0054 \times 10^{7}$ \\
$\mathrm{Ca}$ & $5.1513 \times 10^{5}$ & $2.2313 \times 10^{8}$ & $1.2458 \times 10^{8}$ & $1.3523 \times 10^{7}$ & $7.5504 \times 10^{6}$ \\
\hline
\end{tabular}

References. (1) Goettel (1988).

Table 6. Same as Tables 1 and 2 but for the mineralogical surface composition given by Smith \& Marconi (1995).

\begin{tabular}{cc|cc|cc}
\hline \hline & Cintala & \multicolumn{2}{c}{ Dermott et al. } & \multicolumn{2}{c}{ Wiegert et al. } \\
& & BM-LB & BM-G & BM-LB & BM-G \\
\hline $\mathrm{Na}$ & $7.9026 \times 10^{4}$ & $3.4231 \times 10^{7}$ & $1.9112 \times 10^{7}$ & $2.0746 \times 10^{6}$ & $1.1583 \times 10^{6}$ \\
\hline
\end{tabular}

Notes. $S_{i}$ is given in $N \mathrm{~cm}^{-2} \mathrm{~s}^{-1}$.

References. (1) Smith \& Marconi (1995).

In the discussion below the $\mathrm{Na}, \mathrm{K}, \mathrm{Ca}$ and $\mathrm{Mg}$ neutral atoms are taken into account.

The vapor production rates determined with the $\mathrm{LB}$ and $\mathrm{G}$ calibrations are $6 \pm 2 \times 10^{-13} \mathrm{~g} \mathrm{~cm}^{-2} \mathrm{~s}^{-1}$ and $3.35 \pm 0.67 \times$ $10^{-13} \mathrm{~g} \mathrm{~cm}^{-2} \mathrm{~s}^{-1}$ respectively.

In Tables 4-6 the $S_{i}$ values for the Cremonese et al. (2005), Goettel (1988) and Smith \& Marconi (1995) soil compositions are reported. For a comparison, the $S_{i}$ values computed with the Cintala (1992) estimate of vapor are also reported in the Tables.

Cremonese et al. (2005) give an estimate of Na production rate of $1.8-2.3 \times 10^{6} \mathrm{~N} \mathrm{~cm}^{-2} \mathrm{~s}^{-1}$. The previous tables show that considering the Cremonese et al. (2005) surface composition, the vapor production rate computed assuming most of the dust coming from low eccentricity and low inclination orbits is 96171 times higher than that given by Cremonese et al. (2005). Assuming a much lower fraction of asteroidal dust, as suggested by Wiegert et al. (2009), the vapor production rate is only 711 times higher than that of Cremonese et al. (2005).

If we consider the Goettel surface composition the vapor production rate is 10-20 times higher than that given by Cremonese et al. (2005) or very similar depending from the fraction of asteroidal dust assumed.

It is important to note that in our model all the neutral atoms produced by meteoroid impacts have enough energy to escape Mercury's gravity field. Berezhnoy \& Klumov (2008) estimate that the mass fraction of impact-formed vapor captured by Mercury decreases slightly with increasing impact velocity, from 0.1 at $20 \mathrm{~km} \mathrm{~s}^{-1}$ to 0.07 at $60 \mathrm{~km} \mathrm{~s}^{-1}$. This effect would reduce our estimate for the neutral element production rates of about $10 \%$.

\section{Conclusions}

We estimate the vapor production rate of neutral atoms on Mercury's exosphere considering different calibrations of the dust grain flux and different soil compositions. We adopt the model of Borin et al. (2009) to estimate the micrometeoritic flux on the planets and we tune it to account for different sources of dust. The model is based on the numerical integrations of dust particle trajectories started on low eccentricity and low inclination orbits at the inner edge of the Main Belt. The numerical data of the grain impacts on Mercury are then calibrated using the measured flux of dust on the Earth. However, the facilities measuring the dust flux on Earth (Love \& Brownlee 1993) cannot distinguish between grains coming from low eccentricity and low inclination orbits (possibly of asteroidal origin) and those coming from comets with higher values of the two orbital parameters. For this reason, the flux of dust particles computed by Borin et al. (2009) is tuned with a coefficient that evaluates the source relevance. We consider two different calibrators, one is Love \& Brownlee (1993) and the other is Grün et al. (1985). It is possible to point out that there is a small difference between them in the flux estimate. 
P. Borin et al.: Estimate of the neutral atoms' contribution to the Mercury exosphere caused by a new flux of micrometeoroids

We assumed three simple mineralogical surface compositions for Mercury, finding significant differences in the related production rates. For instance, the production rate of sodium atoms ranges from a minimum of $2.07 \times 10^{6} \mathrm{~N} \mathrm{~cm}^{-2} \mathrm{~s}^{-1}$ up to $3.9 \times 10^{8} \mathrm{~N} \mathrm{~cm}^{-2} \mathrm{~s}^{-1}$. These values are higher than those derived by McClintock et al. (2008) assuming the photon-stimulated desorption as major source. This implies that the impact vaporization appears to have a much higher contribution than previously assumed.

The range of production rates reported in this work is quite wide depending on important parameters that future space missions to Mercury may allow to be better determined. However, thanks to its flexibility our method still could be used to provide accurate estimates.

\section{References}

Berezhnoy, A. A., \& Klumov, B. A. 2008, Icarus, 195, 511

Bida, T. A., Killen, R. M., \& Morgan, T. H. 2000, Nature, 404, 159

Blewett, D. T., Hawke, B. R., \& Lucey, P. G. 2002, Meteorit. Planet. Sci., 37, 1245

Borin, P., Cremonese, G., Marzari, F., Bruno, M., \& Marchi, S. 2009, A\&A, 503, 259

Bruno, M., Cremonese, G., \& Marchi, S. 2007, Planetary and Space Science, 55, 1494

Cintala, M. J. 1992, in J. Geophys. Res., 97

Cremonese, G., Bruno, M., Mangano, V., Marchi, S., \& Milillo, A. 2005, Icarus, 177,122

Dermott, S. F., et al. 2002, Asteroidal dust, in Asteroids III, ed. Bottke, Cellino, Paolicchi, \& Binzel (Arizona University Press)
Goettel, K. A. 1988, Mercury (Tucson, AZ: University of Arizona Press), 613 Grün, E., Zook, H. A., Fechtig, H., \& Giese, R. H. 1985, Icarus, 62, 244 Hunten, D. M., et al. 1988, The Mercury atmosphere, ed. F. Vilas, C. R. Chapman, \& M. S. Matthews (Tucson, AZ: Mercury. Univ. of Arizona Press), 562

Killen, R. M., \& Ip, W. H. 1999, Rev. Geophys., 37, 361

Killen, R. M., Potter, A. E., Reiff, P., et al. 2001, J. Geophys. Res., 106, 20509

Killen, R. M., Bida, T. A., \& Morgan, T. H. 2005, Icarus, 173, 300

Killen, R. M., Bida, T. A., Potter, A. E., et al. 2009, Metallic Species in Mercury's Exosphere Observed by MESSENGER, the Keck I and McMathPierce Telescopes, American Astronomical Society, DPS meeting 2009

Leblanc, F., \& Johnson, R. E. 2003, Icarus, 164, 261

Love, S. G., \& Brownlee, D. E. 1993, Science, 262

Mangano, V., Milillo, A., Mura, A., et al. 2007, Planetary and Space Science, 55, 1541

Mann, I. 2004, Space Sci. Rev., 110, 269

Marchi, S., Morbidelli, A., \& Cremonese, G. 2005, A\&A, 431, 1123

Marzari, F., \& Vanzani, V. 1994, A\&A, 283, 275

McClintock, W. E., Izenberg, N. R., Holsclaw, G. M., et al. 2008, Science, 321, 62

McClintock, W. E., Vervack, R. J., Bradley, E. T., et al. 2009, Science, 324, 610 Morgan, T. H., \& Killen, R. M. 1997, Planet. Space Sci., 45, 81

Morgan, T. H., Zook, H. A., \& Potter, A. E. 1988, Icarus, 75, 156

Potter, A. E. 1995, Geophys. Res. Lett., 22, 3289

Potter, A. E., \& Morgan, T. H. 1985, Science, 229, 651

Potter, A. E., \& Morgan, T. H. 1986, Icarus, 67, 336

Smith, W. H., \& Marconi, M. L. 1995, ApJ, 443, 371

Sprague, A. L., Deutsch, L. K., Hora, J., et al. 2000, Icarus, 147, 421

Sprague, A. L., Emery, J. P., Donaldson, K. L., et al. 2002, Meteorit. Planet. Sci., 37,1255

Sprague, A. L., Donaldson Hanna, K. L., Kozlowski, R. W. H., et al. 2009, Planetary Space Sci., 57, 364

Warell, J. 2003, Icarus, 161, 199

Wiegert, P. 2009, Icarus, 201, 295 